\title{
Asset price fluctuations without aggregate shocks
}

\author{
Costas Azariadis ${ }^{\mathrm{a}}$, Leo Kaas ${ }^{\mathrm{b}, *}$ \\ a Department of Economics, University of California, Los Angeles, USA \\ ${ }^{\mathrm{b}}$ Department of Economics, University of Konstanz, 78457 Konstanz, Germany
}

\begin{abstract}
We analyze the pricing of a productive asset in a class of dynamic exchange economies with heterogeneous, infinitely-lived agents, and self-enforcing intertemporal trades. Individual incomes fluctuate and are correlated; preferences, dividends and aggregate income are fixed. Almost all economies in this class have a unique stationary Markovian equilibrium with fluctuations in asset prices. As the set of unrationed households changes over time and states, excess demand functions shift, asset returns fluctuate, and some households are shut out of asset markets. Examples suggest that the amplitude of these movements is negatively correlated with the productivity of the asset and with the penalty for default.
\end{abstract}

JEL classification: D31; D51; G12

Keywords: Asset prices; Limited commitment; Debt constraints

\section{Introduction}

Asset price volatility is an enduring feature of financial time series and a striking anomaly for dynamic general equilibrium models of asset pricing with homogeneous consumers. Campbell [3], for instance, finds that diversified stock portfolios undergo large movements in returns relative to observed changes in the growth rates of dividends and aggregate consumption. ${ }^{1}$ The puzzling nature of the co-movement between aggregate consumption growth and the price-dividend ratio has led one group of researchers to non-standard assumptions about the representative house-

\footnotetext{
* Corresponding author. Fax: +497531884558.

E-mail address: leo.kaas@uni-konstanz.de (L. Kaas).

${ }^{1}$ Campbell reports in quarterly US data over the period 1947.2 to 1998.4 annualized standard deviations of $15.6 \%$ for real stock returns, $6 \%$ for real dividend growth, and $1.1 \%$ for the seasonally adjusted growth rate in real consumption of non-durables and services.
} 
hold's utility function like strong habit persistence (see [4,5]) and other forms of extremely poor substitutability of consumption at different date-state events.

Another line of research $[6,14]$ segments households in two mutually exclusive groups of active asset market traders and non-participants. Asset returns reflect the characteristics of the first group of stockholding middle-aged and older households, that is, an income profile with declining trend, high mean and high variance. Stockholders bear all equity risk which explains why they demand a low return from safe assets, and their higher consumption variability is consistent with a more substantial equity premium than is possible in economies with homogeneous agents. To understand why the price-to-earnings ratio fluctuates so much, we propose to make the active asset trading group endogenous by endowing households with default-deterring short sales constraints. Asset returns in our model reflect the influence of a changing group of households. Membership in this group adds a new element of risk to the stochastic discount factors that map dividends into asset prices.

Using standard assumptions on preferences, this paper analyzes a class of exchange economies with heterogeneous households and no commitment to intertemporal trades in which the price of a productive asset fluctuates even though the fundamental structure of the economy remains unchanged. This class of economies is the two-state-of-nature counterpart of settings studied in [1] and [10], but with substantially more heterogeneity added. We abstract from any aggregate risk, but focus instead on environments with correlated individual incomes, constant dividends and constant aggregate income. These environments may be interpreted as suffering from asymmetric sectoral shocks which take income away from a few sectors or households and redistribute it to all other sectors or households.

We analyze and compare two related concepts of equilibrium that are based on different assumptions about the enforcement technology. On the one hand, like Kehoe and Levine [9], we consider an equilibrium with limited commitment where consumers are dissuaded from default by a central credit agency that seizes the assets of defaulters and denies them credit for the rest of their lives. As in [1], the threat of market exclusion defines endogenous, agent-specific shortsale constraints that are sufficient to prevent everyone from defaulting. On the other hand, we explore an equilibrium concept of Lustig [13] where defaulters lose their assets in the period of default but keep on trading in asset markets thereafter. With this default technology, consumers can sell securities short up to the value of their capital collateral, effectively facing non-negativity constraints on their net asset positions. For this reason, we refer to an equilibrium with zero debt constraints which appears to be the natural extension of the deterministic economy of Bewley [2] to a stochastic environment. This equilibrium is distinctly different from the one of a liquidity constrained economy as discussed by Kehoe and Levine [10]. In Kehoe and Levine's incomplete markets model, agents trade only capital but no Arrow securities. In their setting and in contrast to ours, a stationary Markovian equilibrium fails to exist (see [10, Proposition 7]). ${ }^{2}$ Besides existence, we also prove uniqueness of stationary Markovian equilibria for both enforcement technologies under standard assumptions.

Our paper adds to this literature the following results. First, asset prices fluctuate in the absence of any aggregate risk. In each period, asset returns are determined by the trading plans of

\footnotetext{
2 The reason for this non-existence is that a low-income consumer sells some capital in order to smooth consumption so that he owns a smaller capital share next period. If the consumer happens to have low income again, he must consume less than in the previous period. Thus, an equilibrium in the incomplete markets economy cannot be a function of the current state alone, but must depend on the whole history of state events. In our equilibrium with zero debt constraints, low-income agents enter each period with the same capital holdings which they use as collateral.
} 
unrationed consumers who buy the productive asset and lend out whatever rationed consumers are allowed to borrow. As the set of unrationed households changes from one period to the next, excess demand correspondences for loans and productive assets shift, causing movements in asset prices. In rough terms, the market applies to future dividends a time-varying discount rate that reflects the marginal rate of intertemporal substitution of consumers who happen to be unrationed each period. This time-varying discounting mechanism vanishes only in two non-generic cases in which asset prices and loan yields remain constant. One case applies to economies in which capital is sufficiently productive and all agents share a common, sufficiently low rate of time preference. In this situation, the first-best commitment equilibrium turns out to be an equilibrium in the limited commitment economy, and possibly in the economy with zero debt constraints. Another case is symmetric economies, like the ones studied in [7] and [10], in which consumers are mirror images of each other and where the Markov process governing state shifts has symmetric transition probabilities. Excess demands for assets in symmetric economies do not vary with time since the set of unrationed consumers has the same tastes and resources every period. If these assumptions fail, a changing set of consumers must be rationed each period, and the economy settles down to a stationary Markovian equilibrium with fluctuations in asset prices. As we show in a two-agent example in Section 4, the volatility of asset prices depends negatively on the productivity of the asset. Further, economies with zero debt constraints have more volatile asset prices than economies with limited commitment. Intuitively, agents are less severely constrained, and the stochastic discount factor fluctuates less, when assets are very productive and when enforcement is strong.

Second, heterogeneity among consumers leads to a natural pattern of endogenous market segmentation. Agents with relatively stable incomes or low risk aversion are rationed out of asset markets forever, while all other agents are recurrently constrained but actively engage in asset market trading. In each period, some agents must be unconstrained, however, and it is these agents' marginal rate of intertemporal substitution that defines the pricing kernel and determines asset returns. In this respect, our model differs from the one of [1] which has no productive asset and in which autarky for all consumers is the unique equilibrium when consumers have relatively small income volatility and low risk aversion. In our model, because of the productive asset, the unique stationary Markovian equilibrium has a positive volume of asset trade so that only some, but not all, consumers stay autarkic. Moreover, market segmentation implies that large volatility in asset returns may go hand in hand with a low volatility of aggregate consumption growth: consumers who do not trade in asset markets have relatively low volatility of income and consumption, but their presence makes no impact on asset prices.

There is a large literature on the role of heterogeneity in asset pricing that is divided into two strands. One assumes that investors have homogeneous preferences but are subject to different idiosyncratic income risks (see [7,8]). When the aggregate state changes, the wealth distribution of investors shifts which causes the set of constrained and unconstrained agents to vary over time. Using a market friction like incomplete markets [7] or trading costs [8], these papers study symmetric economies in which the income distribution only depends on the aggregate state, but is invariant if aggregates are constant. They reach this conclusion either by assuming a two-agent framework in which individual incomes are perfectly negatively correlated [8] or by invoking the law of large numbers for a continuum of investors whose income shocks are cross-sectionally uncorrelated [7]. With any of these assumptions, asset prices cannot fluctuate without movements in aggregate output and dividends, as they do in our paper.

Another group of papers deal with heterogeneity in preferences (see $[11,12,15])$. Krusell and Smith [12] use borrowing constraints to keep agents from perfectly insuring themselves against 
idiosyncratic risk, and they allow for a small amount of heterogeneity in discount factors to match the equilibrium wealth distribution to the data. The law of large numbers applies here as well, and leads to a unique invariant wealth distribution in every aggregate state, so that our mechanism is absent from their model. Kiyotaki and Moore [11] use heterogeneity in preferences and technology to segment households into groups of borrowing and lending agents. The interaction between credit limits and asset prices generates damped oscillations around the unique steady state equilibrium. In contrast, our economy has generally no steady state; the unique asymptotic equilibrium is a cycle. Perhaps closest to ours is the idea of Sandroni [15] that asset price fluctuations are induced by time-varying characteristics of traders. In his model, highly impatient agents endowed with capital shares enter the economy stochastically, causing capital prices to fluctuate. Sandroni, however, assumes complete markets and commitment to intertemporal trade; his mechanism would not generate asset price cycles without this exogenous arrival process. By contrast, in our economy of no commitment, the set of constrained and unconstrained agents changes endogenously, as do asset demand and supply. Furthermore, preference heterogeneity is not decisive for the operation of our mechanism. Heterogeneity of endowments or an asymmetry in the Markov process works just as well.

In the remainder of this paper we set up in Section 2 the general environment for a class of stochastic exchange economies with two states of the world. Section 3 characterizes and compares stationary Markovian equilibria for the two enforcement technologies. Section 4 shows how asset prices fluctuate in these economies. Section 5 concludes. Proofs not included in the text are collected in the Appendix.

\section{The environment}

We study an exchange economy in discrete time $t=0,1, \ldots$ populated by a continuum of infinitely lived agents. There is a finite set of agent types $i \in I$ and a unit mass of identical agents of each type $i$, so that we can identify type $i$ with agent $i$. The structure of the economy is stationary in the sense that agents' preferences are time-independent and aggregate consumption possibilities are constant. There are two states of the world, denoted $A$ and $B$, and shifts between states follow a Markov process where the transition probability from $s \in S \equiv\{A, B\}$ to $s^{\prime} \neq s$ is denoted $\pi_{s} . s_{t} \in S$ is the state in period $t$, and $s^{t}=\left(s_{t}, s_{t-1}, \ldots, s_{0}\right) \in S^{t+1}$ is the state history. $\pi\left(s^{t}\right)$ denotes the unconditional probability of history $s^{t}$, and $\pi\left(s^{\tau} \mid s^{t}\right)$ is the probability of state history $s^{\tau}$ conditional on history $s^{t}$ for any $\tau>t$.

There is a single non-durable consumption good in each period and a non-depreciating durable asset (capital) that pays its owner $d>0$ units of the consumption good in every period and state. The stock of capital is normalized to unity. ${ }^{3}$ The (ex-dividend) capital price at the end of each period is $p\left(s^{t}\right)$. Initial capital endowments are $x_{0}^{i}$ satisfying $\sum_{i} x_{0}^{i}=1$. In addition to capital, agents trade a complete set of state-contingent claims. The price of an Arrow security in period $t$ promising to pay one unit if state $s$ prevails in period $t+1$, given the state history $s^{t}$, is denoted $q_{s}\left(s^{t}\right)$. The absence of arbitrage opportunities between state-contingent claims and capital means that $p\left(s^{t}\right)=q_{A}\left(s^{t}\right)\left(p\left(A, s^{t}\right)+d\right)+q_{B}\left(s^{t}\right)\left(p\left(B, s^{t}\right)+d\right)$ for all $s^{t}$.

Agent $i \in I$ has preferences represented by

$$
(1-\beta) \sum_{t=0}^{\infty} \sum_{s^{t}} \beta^{t} \pi\left(s^{t}\right) u_{i}\left(c^{i}\left(s^{t}\right)\right)
$$

\footnotetext{
${ }^{3}$ When $d=0$, the asset can be interpreted as fiat money. We do not discuss this case here.
} 
defined over consumption plans $\left(c^{i}\left(s^{t}\right)\right)_{s^{t}, t \geqslant 0 .} \beta \in(0,1)$ is the common discount factor. We discuss below how results change when agents have different discount factors. The period utility functions $u_{i}$ are assumed to be twice differentiable, strictly increasing and strictly concave.

Each agent's endowment $\left(y_{S}^{i}\right)$ depends on the current state only. For notational convenience, define $I_{A} \subset I$ as the set of agents who have high income in state $A$ (i.e., $y_{A}^{i}>y_{B}^{i}$ for all $i \in I_{A}$ ) and let $I_{B}=I \backslash I_{A}$ be the set of agents who have high income in state $B$ or whose income does not fluctuate. Since endowments of all agents are perfectly correlated, we interpret individual income fluctuations as sectoral shocks which give rise to labor income variations of the households employed in different sectors of the economy, rather than as strictly idiosyncratic shocks to each individual household. We assume that these sectoral shocks offset each other completely so that there are no aggregate fluctuations; the aggregate endowment in every period is a constant, $\sum_{i \in I} y_{s}^{i} \equiv \Omega, s \in S$. Thus, in each period the consumption good is in aggregate supply $\Omega+d$. We summarize the fundamentals of the economy by the list $\mathcal{E}=\left(\mathcal{Y}, \mathcal{X}_{0}, \mathcal{U}, \beta, d, \pi_{A}, \pi_{B}\right)$ where $\mathcal{Y}=\left(y_{s}^{i}\right)_{i \in I, s \in S}, \mathcal{X}_{0}=\left(x_{0}^{i}\right)_{i \in I}$, and $\mathcal{U}=\left(u_{i}\right)_{i \in I}$.

Let $a^{i}\left(s^{t}\right)$ be the net asset position of agent $i$ when state history $s^{t}$ prevails in period $t$. $a^{i}\left(s^{t}\right)$ may also be written as $\left(p\left(s^{t}\right)+d\right) x^{i}\left(s^{t}\right)+z^{i}\left(s^{t}\right)$ where $x^{i}\left(s^{t}\right)$ are the agent's capital holdings, and $z^{i}\left(s^{t}\right)$ are claims on other agents. Agents face the sequence of budget constraints:

$$
c^{i}\left(s^{t}\right)+q_{A}\left(s^{t}\right) a^{i}\left(A, s^{t}\right)+q_{B}\left(s^{t}\right) a^{i}\left(B, s^{t}\right) \leqslant y_{s_{t}}^{i}+a^{i}\left(s^{t}\right), \quad s^{t} \in S^{t+1}, \quad t \geqslant 0,
$$

where $a^{i}\left(s^{0}\right)=\left(p\left(s^{0}\right)+d\right) x_{0}^{i}+z^{i}\left(s^{0}\right), x_{0}^{i}$ are initial capital holdings, and $z^{i}\left(s^{0}\right)$ are initial state-contingent claims.

A crucial feature of the model is that labor income (endowments) cannot be collateralized so that agents cannot borrow against their future income. We compare two related equilibrium concepts that have been discussed in previous literature. Both concepts specify endogenous constraints $a^{i}\left(s^{t}\right) \geqslant-b^{i}\left(s^{t}\right)$ on the agents' net asset holdings that prevent default at any history $s^{t}$. The first is an equilibrium with limited commitment $[9,1]$ where an enforcement technology permits the exclusion of defaulters from all future trading in asset markets. Endogenous debt constraints are determined to be the maximal values deterring default at any date-event pair. In other words, the debt limit $b^{i}\left(s^{t}\right)$ makes sure that debtor $i$ prefers solvency over default at $s^{t}$, and the constraint binds whenever the debtor is indifferent between these two options. These features are specified in parts (iii) and (iv) of the following definition.

Definition 1. An equilibrium with limited commitment for the economy $\mathcal{E}=\left(Y, X_{0}, \mathcal{U}, \beta, d, \pi_{A}\right.$, $\left.\pi_{B}\right)$ is a list of consumption plans, asset holdings and debt constraints $\left(c^{i}\left(s^{t}\right), a^{i}\left(s^{t}\right), b^{i}\left(s^{t}\right)\right)_{s^{t}, t \geqslant 0}$, $c^{i}\left(s^{t}\right), b^{i}\left(s^{t}\right) \geqslant 0, i \in I$, and a list of security and capital prices $\left(q_{A}\left(s^{t}\right), q_{B}\left(s^{t}\right), p\left(s^{t}\right)\right)_{s^{t}, t \geqslant 0}$ such that

(i) For all $i \in I,\left(c^{i}\left(s^{t}\right), a^{i}\left(s^{t}\right)\right)_{s^{t}}$ maximizes (1) subject to (2) and to $a^{i}\left(s^{t}\right) \geqslant-b^{i}\left(s^{t}\right)$ for all $s^{t}$ at given prices and constraints.

(ii) Markets clear, i.e., for all $s^{t}$,

$$
\sum_{i \in I} c^{i}\left(s^{t}\right)=\Omega+d \quad \text { and } \quad \sum_{i \in I} a^{i}\left(s^{t}\right)=p\left(s^{t}\right)+d .
$$


(iii) Debt constraints prevent default: for any default date $t \geqslant 1$, state history $s^{t}$, and $i \in I$, the market payoff from $t$ forward is no smaller than the default payoff, that is,

$$
V^{i}\left(s^{t}\right) \equiv(1-\beta) \sum_{\tau \geqslant t} \sum_{s^{\tau}} \beta^{\tau-t} \pi\left(s^{\tau} \mid s^{t}\right) u_{i}\left(c^{i}\left(s^{\tau}\right)\right) \geqslant \bar{V}^{i}\left(s^{t}\right),
$$

where

$$
\bar{V}^{i}\left(s^{t}\right) \equiv(1-\beta) \sum_{\tau \geqslant t} \sum_{s^{\tau}} \beta^{\tau-t} \pi\left(s^{\tau} \mid s^{t}\right) u_{i}\left(y_{s_{\tau}}^{i}\right)
$$

is expected utility from autarky from period $t$ onwards.

(iv) Debt constraints are not too tight, i.e., whenever $a^{i}\left(s^{t}\right)=-b^{i}\left(s^{t}\right)$ binds in problem (i), the participation constraint (3) is satisfied with equality.

Parts (i) and (ii) of this definition are standard in commitment economies where debt limits are defined from the intertemporal budget constraint. Part (iii) rules out default, provided that market participation is selected even if it pays off exactly as much as default. Finally, part (iv) specifies how debt limits are calculated in an environment without commitment by the credit authority which is assumed to possess sufficient knowledge of each agent's tastes, endowments and asset trades. ${ }^{4}$ This definition of equilibrium follows Alvarez and Jermann [1] by employing endogenous default-deterring debt limits in the households' problem instead of the equivalent alternative that uses participation constraints in the household problem, as in Kehoe and Levine [9]. ${ }^{5}$ This way of defining equilibrium makes it easier to exploit the households' Euler equations for the characterization of security prices in Section 3. It also helps to compare this equilibrium to the following, alternative concept.

The default technology in an equilibrium with zero debt constraints cannot exclude defaulters from any intertemporal trade but can merely seize their capital shares in the period of default (see [13]). In this situation, agents collateralize their capital holdings to sell securities short, up to the value of their capital collateral. That is, the net asset position must be non-negative, $a^{i}\left(s^{t}\right) \geqslant 0$.

Definition 2. An equilibrium with zero debt constraints for the economy $\mathcal{E}=\left(Y, X_{0}, \mathcal{U}, \beta, d, \pi_{A}\right.$, $\left.\pi_{B}\right)$ is a list of consumption plans, asset holdings and debt constraints $\left(c^{i}\left(s^{t}\right), a^{i}\left(s^{t}\right), b^{i}\left(s^{t}\right)\right)_{s^{t}, t \geqslant 0}$, $c^{i}\left(s^{t}\right), b^{i}\left(s^{t}\right) \geqslant 0, i \in I$, and a list of security and capital prices $\left(q_{A}\left(s^{t}\right), q_{B}\left(s^{t}\right), p\left(s^{t}\right)\right)_{s^{t}, t \geqslant 0}$ such that

(i) and (ii) are as in Definition 1.

(iii) $b^{i}\left(s^{t}\right)=0$ for all $i \in I$ and $s^{t}$.

\footnotetext{
${ }^{4}$ This definition does not require a priori that agents satisfy their intertemporal budget constraint. However, if the present value of the endowment stream at market prices is finite, agents satisfy their intertemporal budget constraint and, consequently, there are no asset price bubbles (see, e.g., [16]).

${ }^{5}$ Since constraints, unlike security prices, are agent-specific, one may wonder whether agents can affect future constraints strategically by changing their behavior today. Such manipulation is, however, excluded in the equilibrium definition: constraints depend only on the agent's incentive to default in any given period, and these incentives are unaffected by what the agent did in previous periods.
} 


\section{Stationary Markovian equilibria}

In this section we characterize stationary Markovian equilibria for both enforcement technologies. A stationary Markovian equilibrium is an allocation of consumption $\left(c_{A}^{i}, c_{B}^{i}\right)_{i \in I}$ and of net asset holdings $\left(a_{A}^{i}, a_{B}^{i}\right)_{i \in I}$ together with four security prices $\left(q_{s^{\prime} s}\right)_{s^{\prime}, s \in S}$ that constitute an equilibrium for an appropriate initial distribution of capital and state-contingent claims. Here $q_{s^{\prime} s}$ is the price of security $s^{\prime} s$ which is traded in state $s$ and promises to pay one unit if state $s^{\prime}$ prevails next period.

Because all agents have the same discount factor, no agent is constrained in his trade of securities $A A$ and $B B$; their prices fall out of the Euler equations as

$$
q_{A A}=\beta\left(1-\pi_{A}\right), \quad q_{B B}=\beta\left(1-\pi_{B}\right) .
$$

Consider agent $i$ who may be constrained in his trade of securities $A B$ and $B A$. The agent's Euler conditions are

$$
\begin{aligned}
& u_{i}^{\prime}\left(c_{B}^{i}\right) q_{A B} \geqslant \beta \pi_{B} u_{i}^{\prime}\left(c_{A}^{i}\right), \\
& u_{i}^{\prime}\left(c_{A}^{i}\right) q_{B A} \geqslant \beta \pi_{A} u_{i}^{\prime}\left(c_{B}^{i}\right) .
\end{aligned}
$$

Each of these inequalities is strict whenever the agent is constrained. Both inequalities together imply that

$$
q_{A B} q_{B A} \geqslant \beta^{2} \pi_{A} \pi_{B} .
$$

Because this inequality is independent of the agent type $i$, all agents $j \neq i$ must be unconstrained whenever $i$ is unconstrained, and all agents $j \neq i$ must be constrained in some state whenever $i$ is. Thus there cannot be equilibria where some agents are constrained and others not. Clearly, this observation is a consequence of the assumption that all agents have the same discount factor.

Consider first the equilibrium with limited commitment and suppose that all agents are constrained when they have low income. Agent $i \in I_{A}$ has low income in state $B$, so his first Euler condition (5) is satisfied with strict inequality. In the economy with limited commitment, this can only be the case when agent $i$ is indifferent in state $A$ between solvency and default. The utility of the solvent agent $i$ in state $A$, who consumes $\left(c_{A}^{i}, c_{B}^{i}\right)$ in all periods, is 6

$$
V_{A}^{i} \equiv \frac{1-\beta\left(1-\pi_{B}\right)}{1-\beta\left(1-\pi_{A}-\pi_{B}\right)}\left(u_{i}\left(c_{A}^{i}\right)+\frac{\beta \pi_{A}}{1-\beta\left(1-\pi_{B}\right)} u_{i}\left(c_{B}^{i}\right)\right) .
$$

Using a similar expression for the defaulting agent who reverts to autarky shows that agent $i$ is indifferent between defaulting and not defaulting if, and only if,

$$
u_{i}\left(c_{A}^{i}\right)+\frac{\beta \pi_{A}}{1-\beta\left(1-\pi_{B}\right)} u_{i}\left(c_{B}^{i}\right)=u_{i}\left(y_{A}^{i}\right)+\frac{\beta \pi_{A}}{1-\beta\left(1-\pi_{B}\right)} u_{i}\left(y_{B}^{i}\right) .
$$

Agent $i \in I_{A}$ may be constrained or unconstrained in his high-income state $A$. If the agent is unconstrained in state $A$, the second Euler condition (6) holds with equality. Agent $i$ 's consumption levels, to be denoted $c_{A}^{i}\left(q_{B A}\right)$ and $c_{B}^{i}\left(q_{B A}\right)$ are implicitly defined by the binding Euler equation (6) and the binding participation constraint (9). Geometrically, they parameterize an indifference curve of agent $i$ in $\left(c_{A}^{i}, c_{B}^{i}\right)$ space. The function $c_{A}^{i}(.) \leqslant y_{A}^{i}$ is strictly increasing in $q_{B A}$, and

\footnotetext{
${ }^{6}$ The formula is derived by solving two recursive equations in two unknowns which are the stationary utility levels of agent $i$ in the two states.
} 
$c_{B}^{i}(.) \geqslant y_{B}^{i}$ is strictly decreasing, because agent $i$ wants to transfer less wealth from state $A$ to state $B$ when the security price $q_{B A}$ is higher. When $q_{B A}$ exceeds the reservation price $\bar{q}_{B A}^{i} \equiv$ $\beta \pi_{A} u_{i}^{\prime}\left(y_{B}^{i}\right) / u_{i}^{\prime}\left(y_{A}^{i}\right)$, however, agent $i$ does not want to buy any securities in order to shift income from state $A$ to state $B$ and instead prefers to go short in his trade of security $B A$. But then agent $i$ must be constrained in both states of the world, and the binding participation constraints imply that the agent is autarkic. Thus agent $i$ 's consumption satisfies $c_{A}^{i}\left(q_{B A}\right)=y_{A}^{i}$ and $c_{B}^{i}\left(q_{B A}\right)=y_{B}^{i}$ for all $q_{B A} \geqslant \bar{q}_{B A}^{i}$.

Summing over all agents $i \in I_{A}$ yields "aggregate excess demand functions" of the group $I_{A}$ in both states of the world as follows:

$$
Z_{A}^{A}\left(q_{B A}\right) \equiv \sum_{i \in I_{A}}\left(c_{A}^{i}\left(q_{B A}\right)-y_{A}^{i}\right)(\leqslant 0), \quad Z_{B}^{A}\left(q_{B A}\right) \equiv \sum_{i \in I_{A}}\left(c_{B}^{i}\left(q_{B A}\right)-y_{B}^{i}\right)(\geqslant 0) .
$$

Similarly, we can define excess demand functions for agents in group $I_{B}$ which now depend only on the security price $q_{A B}$ :

$$
Z_{A}^{B}\left(q_{A B}\right) \equiv \sum_{i \in I_{B}}\left(c_{A}^{i}\left(q_{A B}\right)-y_{A}^{i}\right)(\geqslant 0), \quad Z_{B}^{B}\left(q_{A B}\right) \equiv \sum_{i \in I_{B}}\left(c_{B}^{i}\left(q_{A B}\right)-y_{B}^{i}\right)(\leqslant 0) .
$$

A stationary Markovian equilibrium with constrained agents is characterized by security prices $\left(q_{A B}, q_{B A}\right)$ satisfying (7) and the following pair of market-clearing equations in both states of the world:

$$
\begin{aligned}
& Z_{A}^{A}\left(q_{B A}\right)+Z_{A}^{B}\left(q_{A B}\right)=d, \\
& Z_{B}^{A}\left(q_{B A}\right)+Z_{B}^{B}\left(q_{A B}\right)=d .
\end{aligned}
$$

In the Appendix we show that this pair of equations can have at most one solution satisfying (7). Whenever there is no solution satisfying (7), there must exist a first-best stationary Markovian equilibrium with perfect risk sharing. A necessary and sufficient condition for such an equilibrium is obtained as follows. Let $\bar{c}^{i}$ be the flat consumption level at which agent $i \in I_{A}$ is indifferent between default and solvency in state $A$. Using (9), this consumption level satisfies

$$
u_{i}\left(\bar{c}^{i}\right)=\frac{1-\beta\left(1-\pi_{B}\right)}{1-\beta\left(1-\pi_{A}-\pi_{B}\right)} u_{i}\left(y_{A}^{i}\right)+\frac{\beta \pi_{A}}{1-\beta\left(1-\pi_{A}-\pi_{B}\right)} u_{i}\left(y_{B}^{i}\right) .
$$

Similarly consumption levels for agents in group $I_{B}$ are defined. The consumption profile $\left(\bar{c}^{i}\right)_{i \in I}$ is the minimum allocation of flat consumption that prevents default of all agents. This allocation is feasible if

$$
\sum_{i \in I} \bar{c}^{i} \leqslant \Omega+d
$$

This condition is necessary and sufficient for the first best to be an equilibrium with limited commitment for some initial distribution of wealth. It is satisfied if the common discount factor $\beta$ is sufficiently large, if the dividend is sufficiently large, or if agents are sufficiently risk-averse. The size of income variability has an ambiguous effect, however: perfect risk sharing is an equilibrium either for small or for large variability. ${ }^{7}$ With small fluctuations, agents can smooth consumption perfectly by trading capital without the need to borrow. With large fluctuations, agents borrow

\footnotetext{
${ }^{7}$ Formally, $\bar{c}^{i}$ is first increasing and then decreasing in the variance of $y_{s}^{i}$.
} 
but they do not default because asset market exclusion is too severe a punishment for them. In the Appendix we show ${ }^{8}$

Proposition 1. There exists a unique stationary Markovian equilibrium with limited commitment in which either,

(i) condition (14) holds and all agents are unconstrained in both states of the world, or,

(ii) condition (14) does not hold and all agents are constrained in their low-income state. Some agents may not trade any assets and may remain autarkic.

Consider now the economy with zero debt constraints, and suppose again that agent $i \in I_{A}$ is constrained in his trade of security $A B$. With zero debt constraints, this means that the agent starts any high-income state $A$ with zero net asset holdings so that $a_{A}^{i}=0$. The two budget constraints in states $A$ and $B$ are $c_{A}^{i}+q_{B A} a_{B}^{i}=y_{A}^{i}$ and $c_{B}^{i}+q_{B B} a_{B}^{i}=y_{B}^{i}+a_{B}^{i}$. Together with (4) these equations yield a single intertemporal budget constraint,

$$
\left(1-\beta\left(1-\pi_{B}\right)\right)\left(y_{A}^{i}-c_{A}^{i}\right)=q_{B A}\left(c_{B}^{i}-y_{B}^{i}\right) .
$$

As above, agent $i$ is either unconstrained in his trade of security $B A$, or he stays autarkic. In the first case, the agent transfers wealth from his high-income state $A$ to state $B$ by buying capital shares in state $A$ and selling them short against next period's state $A$ (since $a_{A}^{i}=0$ ). His consumption, denoted again as $c_{A}^{i}\left(q_{B A}\right)$ and $c_{B}^{i}\left(q_{B A}\right)$, is then implicitly determined from the binding second Euler equation (6) and the zero-debt condition (15). These equations correspond to the offer curve of a (fictitious) two-period utility-maximization problem of an agent with preferences (8) and income-profile $\left(y_{A}^{i}, y_{B}^{i}\right)$. Under the assumption that consumption goods in the two periods are gross substitutes, $c_{A}^{i}($.$) is decreasing and c_{B}^{i}$ (.) is increasing. As before, agent $i$ remains autarkic when $q_{B A} \geqslant \bar{q}_{B A}^{i}$ and then $c_{s}^{i}\left(q_{B A}\right)=y_{s}^{i}, s=A, B$. By summing over the agents' individual excess demands, aggregate excess demand curves are defined as in (10) with the only difference that they are obtained from parameterizations of offer curves rather than indifference curves, as before. A stationary Markovian equilibrium with zero debt constraints is again a solution to Eqs. (11) and (12) which turns out to be unique under the assumption of gross substitutes (see Appendix).

When is risk sharing perfect in the equilibrium with zero debt constraints? The minimum flat consumption of agent $i \in I_{A}$ that is compatible with non-negative asset positions, to be denoted $\bar{c}^{i}$, follows from (15) and $q_{B A}=\beta \pi_{A}$ as

$$
\bar{c}^{i}=\frac{1-\beta\left(1-\pi_{B}\right)}{1-\beta\left(1-\pi_{A}-\pi_{B}\right)} y_{A}^{i}+\frac{\beta \pi_{A}}{1-\beta\left(1-\pi_{A}-\pi_{B}\right)} y_{B}^{i} .
$$

There exists a first-best allocation which is a stationary Markovian equilibrium for some distribution of initial wealth whenever the feasibility condition $\sum_{i \in I} \bar{c}^{i} \leqslant \Omega+d$ is satisfied. Manipulation

\footnotetext{
${ }^{8}$ It is important to mention that perfect risk-sharing can never be an equilibrium when agents have different discount factors. In the first best, consumption of impatient agents tends to zero in the long run, so that these agents necessarily violate their participation constraint at some future date. Thus, in a larger class of economies that allows for different discount factors, the first-best is almost never an equilibrium. Put differently, binding constraints and fluctuations in asset prices are a generic phenomenon.
} 
of this condition gives

$$
\sum_{i \in I}\left|y_{A}^{i}-y_{B}^{i}\right| \leqslant \frac{2 d\left(1-\beta+\beta\left(\pi_{A}+\pi_{B}\right)\right)}{1-\beta} .
$$

This condition is necessary and sufficient for the existence of a first-best equilibrium with zero debt constraints. As before, productive capital and patient consumers are conducive for perfect risk sharing. But in contrast to the economy with perfect risk sharing, the degree of risk aversion is irrelevant, and large enough income fluctuations unambiguously lead to binding debt constraints.

A comparison of (13) and (16) shows that $\bar{c}^{i}$ is larger in the economy with zero debt constraints than in the economy with limited commitment since $u_{i}$ is strictly concave. Hence, (17) is stronger than (14), and the two conditions fall together in the limit of risk neutrality. Intuitively, when agents are risk neutral they are not punished by the exclusion from asset market trading. Therefore, they must face zero debt constraints even in the economy with limited commitment. In the Appendix we prove

Proposition 2. Suppose that intertemporal consumption goods are gross substitutes. Then there exists a unique stationary Markovian equilibrium with zero debt constraints in which either,

(i) condition (17) holds and all agents are unconstrained in both states of the world, or,

(ii) condition (17) does not hold and all agents are constrained in their low-income state. Some agents may not trade any assets and may remain autarkic.

Propositions 1 and 2 show that the two equilibrium concepts have rather similar features. They both give rise to endogenous market segmentation: some agents with low income variability or low risk aversion do not trade securities, just consuming their endowments; other agents with larger income variability or higher risk aversion actively trade in asset markets. Furthermore, when all agents have the same rate of time preference, either all agents are unconstrained or all agents are constrained in their low-income state of the world.

\section{Asset price fluctuations}

We show now that the capital price fluctuates in every constrained equilibrium for almost all economies. From the no-arbitrage conditions, one obtains capital prices as functions of security prices:

$$
\begin{aligned}
& p_{A}=\frac{q_{A A}\left(1-q_{B B}\right)+q_{B A}\left(1+q_{A B}\right)}{\left(1-q_{A A}\right)\left(1-q_{B B}\right)-q_{B A} q_{A B}} d, \\
& p_{B}=\frac{q_{B B}\left(1-q_{A A}\right)+q_{A B}\left(1+q_{B A}\right)}{\left(1-q_{A A}\right)\left(1-q_{B B}\right)-q_{B A} q_{A B}} d .
\end{aligned}
$$

Thus, the capital price does not fluctuate if, and only if,

$$
q_{A A}+q_{B A}=q_{B B}+q_{A B} .
$$

In the first-best equilibrium, this equality is trivially fulfilled. In a constrained equilibrium, two of these security prices are given in (4), and the other two security prices are the unique solution to the pair of Eqs. (11) and (12). Nothing, however, guarantees that this solution satisfies the restriction (20). Indeed, (20) is violated for almost all economies in our class. The only important exception are symmetric economies (such as the one studied by Kehoe and Levine [10]). In such 
economies, agents in groups $I_{A}$ and $I_{B}$ are "mirror images" of each other with the same utility functions, discount factors and income profiles so that their individual income fluctuations are exactly offsetting, and transition probabilities between the two states are equal, $\pi_{A}=\pi_{B}=\pi$. If these conditions are fulfilled, $Z_{A}^{A}=Z_{B}^{B}$ and $Z_{A}^{B}=Z_{B}^{A}$, and (11) and (12) imply that $q_{B A}=q_{A B}$. Since also $q_{A A}=q_{B B}=\beta(1-\pi)$, Eq. (20) holds and the capital price does not fluctuate.

To illustrate how heterogeneity affects asset price fluctuations and to explore the impact of economic fundamentals on the size of these fluctuations, we discuss an example of an economy with symmetric endowments and preferences, and an asymmetry in the transition probabilities between states. ${ }^{9}$ Suppose there are two agents $i=1,2$ with identical discount factor $\beta$ and period utility function $u$. In state $A$, agent 1 has income $1+\alpha$ and agent 2 has income $1-\alpha$. State $B$ has the reverse income configuration. Capital productivity equals $d>0$ in each state, and aggregate income $\Omega_{d}=2+d$ is state-independent. Transition probabilities are $\pi_{A}$ and $\pi_{B}$. The economy is symmetric whenever $\pi_{A}=\pi_{B}$ in which case there are no fluctuations in asset prices. From the analysis of the previous section, a constrained equilibrium with limited commitment is an allocation $\left(c_{A}^{1}, c_{A}^{2}, c_{B}^{1}, c_{B}^{2}\right)$ satisfying the binding participation constraints:

$$
\begin{aligned}
& u\left(c_{A}^{1}\right)+\frac{\beta \pi_{A}}{1-\beta\left(1-\pi_{B}\right)} u\left(c_{B}^{1}\right)=u(1+\alpha)+\frac{\beta \pi_{A}}{1-\beta\left(1-\pi_{B}\right)} u(1-\alpha), \\
& u\left(c_{B}^{2}\right)+\frac{\beta \pi_{B}}{1-\beta\left(1-\pi_{A}\right)} u\left(c_{A}^{2}\right)=u(1+\alpha)+\frac{\beta \pi_{B}}{1-\beta\left(1-\pi_{A}\right)} u(1-\alpha),
\end{aligned}
$$

plus the market-clearing conditions $c_{A}^{1}+c_{A}^{2}=2+d$ and $c_{B}^{1}+c_{B}^{2}=2+d$. Geometrically, the equilibrium is at the intersection of the indifference curves of the two agents in the Edgeworth-box diagram of Fig. 1 (point E). In the diagram, $\pi_{A}$ is bigger than $\pi_{B}$, so that agent 1 is more likely to have low income than agent 2 . Therefore, agent 1 cares more about the threat of market exclusion which is reflected in a higher "effective discount factor" in the participation constraint (PC1) relative to the one of agent 2 in (PC2). The equilibrium with zero debt constraints has similar features-the only difference is that indifference curves (PC1) and (PC2) are replaced by offer curves corresponding to the utility functions in (PC1) and (PC2).

Because of the difference in effective discount factors, agent 2's indifference curve (offer curve) is steeper than agent 1's indifference curve (offer curve), so that their intersection is above the cross-diagonal that defines symmetric allocations. An equilibrium with rationing requires that agents be constrained in their supply of claims contingent on their high-income state. For agent 1 this means $q_{A B}>\beta \frac{u^{\prime}\left(c_{A}^{1}\right)}{u^{\prime}\left(c_{B}^{1}\right)} \pi_{B}$. Since agent 2 is unconstrained in his demand for claims contingent on his low-income state $A$, we must have that $q_{A B}=\beta \frac{u^{\prime}\left(c_{A}^{2}\right)}{u^{\prime}\left(c_{B}^{2}\right)} \pi_{B}$. From these two conditions we see that agent 1 is constrained if, and only if,

$$
\frac{u^{\prime}\left(c_{B}^{1}\right)}{u^{\prime}\left(c_{A}^{1}\right)}>\frac{u^{\prime}\left(c_{B}^{2}\right)}{u^{\prime}\left(c_{A}^{2}\right)} \text {. }
$$

By a similar argument, agent 2 is constrained in his supply of claims contingent on his high-income state $B$ if, and only if, (21) holds. Hence agent 1 is constrained in some state if, and only if, agent 2 is constrained in the other state. Moreover, market clearing implies that (21) holds if $c_{A}^{1}>c_{B}^{1}$

\footnotetext{
${ }^{9}$ It is not difficult to construct other examples where the asymmetry is in the endowment process or in risk aversion. It is also straightforward to add to this economy an arbitrary number of autarkic consumers with sufficiently low income variability or low risk aversion.
} 


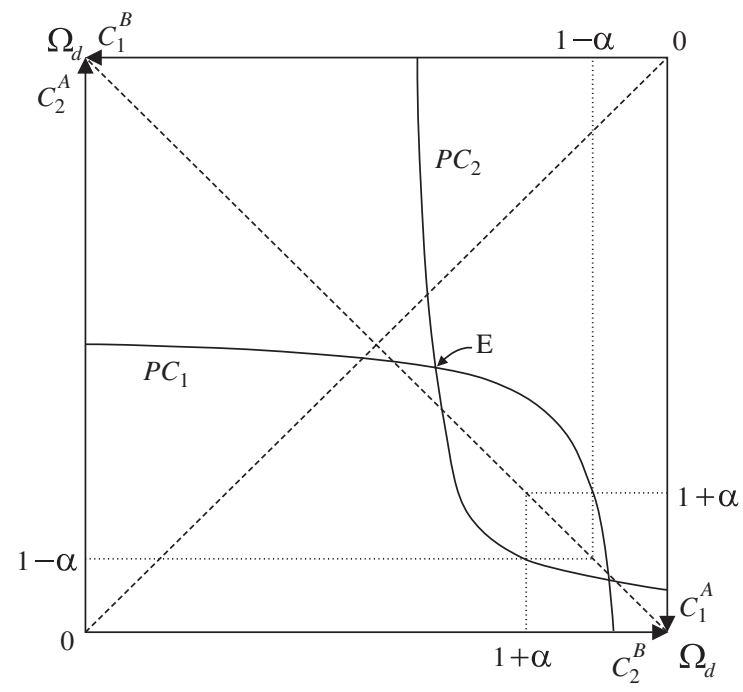

Fig. 1. Equilibrium with limited commitment in a two-agent economy with asymmetric transition probabilities.

(or $c_{A}^{2}<c_{B}^{2}$ ), that is, if agents do not smooth consumption perfectly. Thus, the intersection of the indifference curves (offer curves) must be below the diagonal in Fig. 1. When the curves cut above the diagonal, the unique equilibrium is a first-best equilibrium. This happens, for instance, when $d$ or $\beta$ are large enough. Moreover, implementability of the commitment solution is also favored by less persistent technology shocks which make indifference curves (offer curves) flatter. Intuitively, if the income process is very volatile, agents need the market more so that default is less attractive (cf. [10]).

How much does the capital price fluctuate when the Markov dynamics is asymmetric $\left(\pi_{A}>\pi_{B}\right)$ and agents are constrained? Because $1>c_{A}^{2} / c_{B}^{2}>c_{B}^{1} / c_{A}^{1}$ (see Fig. 1) we have

$$
1<\frac{u^{\prime}\left(c_{A}^{2}\right)}{u^{\prime}\left(c_{B}^{2}\right)}<\frac{u^{\prime}\left(c_{B}^{1}\right)}{u^{\prime}\left(c_{A}^{1}\right)},
$$

and thus $q_{A B}<q_{B A}$ and $q_{A A}<q_{B B}$. Clearly, agent 2 pays a lower price to insure against his low-income state than agent 1 . Inequality (22) yields

$$
q_{A A}+q_{B A}=\beta\left(1+\pi_{A}\left(\frac{u^{\prime}\left(c_{B}^{1}\right)}{u^{\prime}\left(c_{A}^{1}\right)}-1\right)\right)>\beta\left(1+\pi_{B}\left(\frac{u^{\prime}\left(c_{A}^{2}\right)}{u^{\prime}\left(c_{B}^{2}\right)}-1\right)\right)=q_{B B}+q_{A B} .
$$

From (18) and (19), the capital price is lower in state $B$ than in state $A$. Since agent 1 has less persistently high income, he pays a higher price for capital than agent 2 . When the productivity of capital goes up, indifference curves move outwards, the difference between $c_{A}^{2} / c_{B}^{2}$ and $c_{B}^{1} / c_{A}^{1}$ becomes smaller, and so does the difference between $p_{A}$ and $p_{B}$. Hence, asset price volatility should be lower in more productive economies. Furthermore, because constraints are tighter in the economy with zero debt constraints than in the one with limited commitment, one should also expect asset price volatility to be bigger.

Exploring these issues analytically is difficult since closed-form expressions for the capital price cannot be obtained. We therefore performed a numerical experiment with $u(c)=\ln (c)$, 

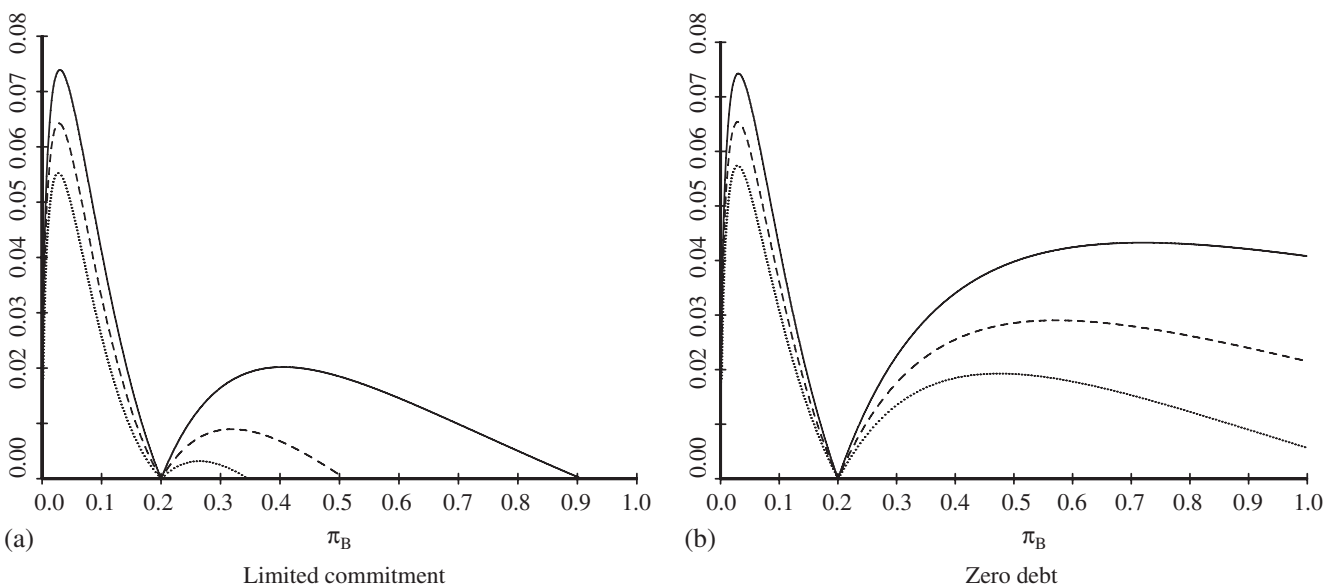

Fig. 2. Relative standard deviation of the capital price for varying $\pi_{B}$ and three levels of productivity $(d=0.01$ solid, $d=0.02$ dashed, $d=0.03$ dotted).

$\beta=0.9, \alpha=0.2$ and $\pi_{A}=0.2$ and we let $\pi_{B}$ vary from zero to unity. We set the dividend at three values $0.01,0.02$ and 0.03 . Fig. 2 shows the relative standard deviation (i.e., standard deviation divided by the mean) of the capital price as the transition probability $\pi_{B}$ varies from zero to one. The symmetric economy $\left(\pi_{B}=0.2\right)$ produces no fluctuations. In all asymmetric economies, volatility is bigger in the economy with zero debt constraints (Fig. 2(b)) than in the one with limited commitment (Fig. 2(a)). The volatility difference between the two economies is almost negligible for values of $\pi_{B}<\pi_{A}=0.2$ but much larger for $\pi_{B}>\pi_{A}=0.2$. The figures also show that fluctuations are smaller in more productive economies. Moreover, in the economy with limited commitment fluctuations disappear when $\pi_{B}$ is big enough in which case risk sharing is perfect. In these situations, agent 1 has persistently high income in state $A$ and is rewarded with high average consumption for buying claims from agent 2 who has persistently low income in state $A$. Both agents have strong incentives to use asset markets; the outcome is a first-best equilibrium with no fluctuations in asset prices.

Finally, it is worth mentioning that if (20) fails, then we have fluctuations not only in the capital price but also in the safe interest rate which varies between the two values $1 /\left(q_{A A}+q_{B A}\right)$ and $1 /\left(q_{B B}+q_{A B}\right)$. Since stock returns are empirically much more volatile than safe returns, a natural question is whether this is also true in our model. We have checked this issue numerically as well. For the same parameter configuration as above, we found that the volatility of the capital return exceeds the volatility of the safe return by roughly a factor of two. The equity premium remains moderate. However, after adding a small amount of aggregate risk to this example with logarithmic preferences, we were able to generate an equity premium that was substantially bigger in asymmetric economies than in the corresponding symmetric economy. Details on these numerical studies can be obtained from the authors upon request.

\section{Conclusions}

We have studied a class of exchange economies with stationary tastes, stationary consumption possibilities and no commitment to intertemporal trades. We compared an equilibrium with 
limited commitment where default is punished by seizure of assets and market exclusion with an equilibrium with zero debt constraints where defaulters lose their asset holdings but cannot be excluded from future market participation. If consumers are sufficiently heterogeneous and have correlated incomes, equilibria in both economies have unusual properties: rationing, market segmentation and, above all, non-stationarity. Most economies in this class have the following features:

1. The stationary equilibrium is a unique stochastic cycle in which yields and asset prices fluctuate while dividends and aggregate consumption are constant. Asset returns at each point in time reflect the marginal rates of substitution of agents who are not rationed at that point. As the set of unrationed agents changes over time, so do asset prices.

2. Rationing occurs every period, and some agents with low income variability or low risk aversion may never engage in intertemporal trades. If all agents have the same discount factor, either all agents are constrained in their bad state, or no agent is.

3. The example in Section 4 suggests that the amplitude of the stochastic cycle in asset prices is negatively correlated with the productivity of assets and with the penalty for default, that is, with the strength of the mechanism that enforces intertemporal trades. Fluctuations are quantitatively larger if assets are unproductive and default is punished lightly.

\section{Acknowledgments}

Support from the Austrian Science Fund (FWF) and the UCLA Faculty Senate are gratefully acknowledged. We would like to thank Rody Manuelli and participants at the 10th Texas Monetary Conference in Austin, the SWET Conference 2002 in San Diego, the NBER General Equilibrium Conference 2002 in Minneapolis, and at seminars at Stanford and at UCSB for helpful comments. Discussions during the conference on "Belief Formation and Fluctuations in Economic and Financial Markets" sponsored by the Volkswagenstiftung are gratefully acknowledged. The usual disclaimer applies.

\section{Appendix A}

Proof of Proposition 1. It is to show that there is a unique solution $\left(q_{A B}, q_{B A}\right)$ to the pair of market-clearing equations

$$
\begin{aligned}
& \text { (MCA) } Z_{A}^{A}\left(q_{B A}\right)+Z_{A}^{B}\left(q_{A B}\right)=d, \\
& (\mathrm{MCB}) \quad Z_{B}^{A}\left(q_{B A}\right)+Z_{B}^{B}\left(q_{A B}\right)=d,
\end{aligned}
$$

satisfying $q_{A B} q_{B A}>\beta^{2} \pi_{A} \pi_{B}$ if, and only if, the first-best solution is not an equilibrium. By definition, $Z_{A}^{A}\left(q_{B A}\right)=\sum_{i \in I_{A}}\left(c_{A}^{i}\left(q_{B A}\right)-y_{H}^{i}\right) \leqslant 0$ is a piecewise differentiable, non-decreasing function which equals zero for all $q_{B A} \geqslant \bar{q}_{B A} \equiv \max _{i \in I_{A}} \bar{q}_{B A}^{i}$ (as defined in the text, $\bar{q}_{B A}^{i}$ is the reservation price of security $B A$ above which agent $i$ remains autarkic). Analogously, $Z_{B}^{B}\left(q_{A B} \leqslant 0\right.$ is a non-decreasing function which equals zero for all $q_{A B} \geqslant \bar{q}_{A B} \equiv \max _{i \in I_{B}} \bar{q}_{A B}^{i}$. On the other hand, the functions $Z_{B}^{A}\left(q_{B A}\right)$ and $Z_{A}^{B}\left(q_{A B}\right)$ are non-negative, non-increasing, and they are equal to zero for $q_{B A} \geqslant \bar{q}_{B A}\left(q_{A B} \geqslant \bar{q}_{A B}\right.$, resp.). Because of these features, each of the two equations (MCA) and (MCB) defines an upward-sloping curve in $\left(q_{A B}, q_{B A}\right)$ space. Because $d>0$, (MCA) leaves the rectangle defined by $q_{A B} \leqslant \bar{q}_{A B}$ and $q_{B A} \leqslant \bar{q}_{B A}$ at some point $q_{A B}<\bar{q}_{A B}$ and $q_{B A}=\bar{q}_{B A}$, whereas (MCB) leaves this rectangle at some point $q_{A B}=\bar{q}_{A B}$ and $q_{B A}<\bar{q}_{B A}$, as shown in Fig. 3(a) and (b). 
The proof proceeds in two steps:

1. We show that (MCA) and (MCB) can have at most one interior solution, $q_{A B} q_{B A}>\beta^{2} \pi_{A} \pi_{B}$.

2. Whenever an interior solution exists, the first-best solution is not an equilibrium with limited commitment, and vice versa.

To prove 1 , suppose that there are two interior solutions at $\left(q_{A B}, q_{B A}\right) \ll\left(\tilde{q}_{A B}, \tilde{q}_{B A}\right)$. Because the curve (MCA) leaves the rectangle bounded by $\bar{q}_{A B}$ and $\bar{q}_{B A}$ to the left of the curve (MCB), (MCA) is flatter than (MCB) at the equilibrium with lower security prices, but steeper at the other equilibrium. Thus, at the equilibrium with lower security prices, (MCA) is flatter than (MCB), if and only if,

$$
\frac{\mathrm{d} q_{B A}}{\mathrm{~d} q_{A B}}\left|(\mathrm{MCA})=-\frac{Z_{A}^{B^{\prime}}}{Z_{A}^{A^{\prime}}}<\frac{\mathrm{d} q_{B A}}{\mathrm{~d} q_{A B}}\right|_{(\mathrm{MCB})}=-\frac{Z_{B}^{B^{\prime}}}{Z_{B}^{A^{\prime}}},
$$

which holds whenever $1>\phi^{A}\left(q_{B A}\right) \phi^{B}\left(q_{A B}\right)$ where

$$
\phi^{A}\left(q_{B A}\right) \equiv-\frac{Z_{B}^{A^{\prime}}\left(q_{B A}\right)}{Z_{A}^{A^{\prime}}\left(q_{B A}\right)}, \quad \phi^{B}\left(q_{A B}\right) \equiv-\frac{Z_{A}^{B^{\prime}}\left(q_{A B}\right)}{Z_{B}^{B^{\prime}}\left(q_{A B}\right)} .
$$

Because (MCA) is steeper than (MCB) at the equilibrium with higher security prices, we obtain by similar reasoning $\phi^{A}\left(\tilde{q}_{B A}\right) \phi^{B}\left(\tilde{q}_{A B}\right)>1$. Lemma 1 below shows, however, that $\phi^{A}$ (and so also $\phi^{B}$ ) are decreasing functions which contradicts the above starting point of two interior solutions satisfying $q_{A B}<\tilde{q}_{A B}, q_{B A}<\tilde{q}_{B A}$.

To show 2, suppose first that the first-best solution with $q_{B A}^{*}=\beta \pi_{A}$ and $q_{A B}^{*}=\beta \pi_{B}$ is an equilibrium. Note again that $Z_{A}^{A}\left(q_{B A}^{*}\right)$ and $Z_{B}^{A}\left(q_{B A}^{*}\right)$ are excess demands of group $I_{A}$ in states $A$ and $B$ with the feature that the participation constraints for the agents in this group are satisfied with equality. Thus, the first-best solution, when it is an equilibrium with limited commitment, must promise these agents at least as high consumption levels in both states to guarantee that their participation constraint is satisfied, and the same holds for the agents in group $I_{B}$. Therefore,

$$
Z_{A}^{A}\left(q_{B A}^{*}\right)+Z_{A}^{B}\left(q_{A B}^{*}\right) \leqslant d, \quad Z_{B}^{A}\left(q_{B A}^{*}\right)+Z_{B}^{B}\left(q_{A B}^{*}\right) \leqslant d .
$$

Hence there is an "excess supply" in both states at the price vector $\left(q_{A B}^{*}, q_{B A}^{*}\right)$. However, an excess supply in state $A$ is to the right of the curve (MCA), whereas an excess supply in state $B$ is to the left of the curve (MCB) in Fig. 3. Consequently, (MCA) cuts the curve $q_{A B} q_{B A}=\beta^{2} \pi_{A} \pi_{B}$ to the left of (MCB), as in Fig. 3(a). Hence, there cannot be an interior intersection between these two curves: if there was such an intersection, there must be at least one further intersection, but this has been ruled out above.

Conversely, suppose now that no first-best allocation is an equilibrium so that the participation constraint of some agent must be violated at any consumption vector satisfying the first-order conditions at the price vector $\left(q_{A B}^{*}, q_{B A}^{*}\right)$. Therefore, at this price vector, there must be an excess demand in both states $A$ and in state $B$ because the excess demand curves $Z_{A}^{A}$, etc. reflect the minimum consumption that is needed to satisfy all agents' participation constraints:

$$
Z_{A}^{A}\left(q_{B A}^{*}\right)+Z_{A}^{B}\left(q_{A B}^{*}\right)>d, \quad Z_{B}^{A}\left(q_{B A}^{*}\right)+Z_{B}^{B}\left(q_{A B}^{*}\right)>d .
$$

By the same reasoning as above, (MCA) cuts the curve $q_{A B} q_{B A}=\beta^{2} \pi_{A} \pi_{B}$ to the right of (MCB), as shown in Fig. 3(b). Because of the boundary behavior of these curves shown above, there must 


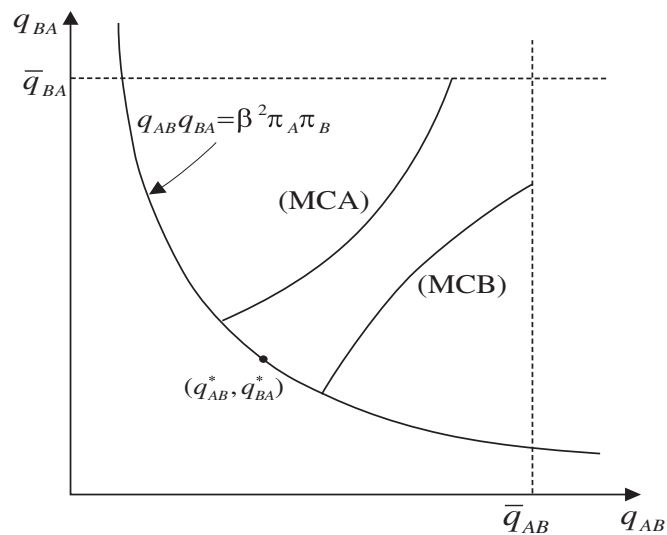

(a)

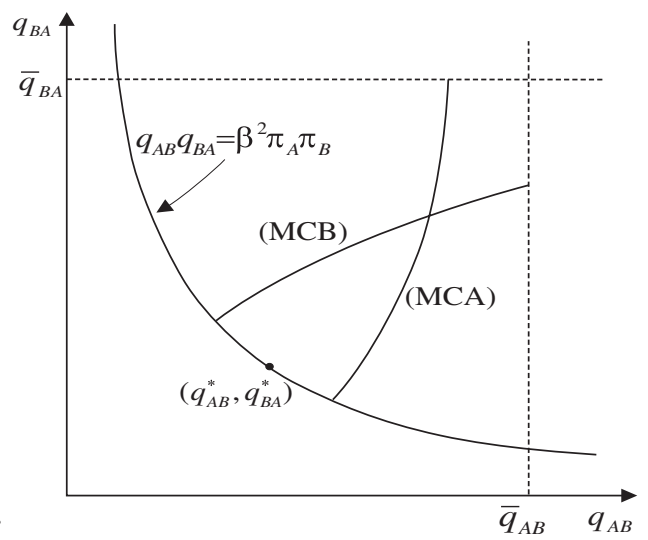

(b)

Fig. 3. Stationary Markovian equilibrium: (a) commitment equilibrium; (b) constrained equilibrium.

be an intersection which is the unique stationary Markovian equilibrium. This completes the proof.

Lemma 1. $\phi^{A}\left(q_{B A}\right)=-\frac{Z_{B}^{A^{\prime}}\left(q_{B A}\right)}{Z_{A}^{A^{\prime}}\left(q_{B A}\right)}$ is decreasing.

Proof of Lemma 1. We drop the superindex $A$ from all calculations because only agents from group $I_{A}$ are involved. The function $\phi$ is decreasing iff $Z_{B}^{\prime} Z_{A}^{\prime \prime}<Z_{B}^{\prime \prime} Z_{A}^{\prime}$ which is equivalent to

$$
\frac{Z_{A}^{\prime \prime} q_{B A}}{Z_{A}^{\prime}}>\frac{Z_{B}^{\prime \prime} q_{B A}}{Z_{B}^{\prime}}
$$

To show (24) we need another Lemma.

Lemma 2. Consider the individual consumption demands $\left(c_{A}^{i}\left(q_{B A}\right), c_{B}^{i}\left(q_{B A}\right)\right)$ as implicitly defined by the binding participation constraint and the Euler equation:

$$
\begin{aligned}
u\left(c_{A}^{i}\right)+\frac{\beta \pi_{A}}{1-\beta\left(1-\pi_{B}\right)} u\left(c_{B}^{i}\right) & =u\left(y_{A}^{i}\right)+\frac{\beta \pi_{A}}{1-\beta\left(1-\pi_{B}\right)} u\left(y_{B}^{i}\right), \\
q_{B} u_{i}^{\prime}\left(c_{A}^{i}\right) & =\beta \pi_{A} u_{i}^{\prime}\left(c_{B}^{i}\right) .
\end{aligned}
$$

These demand curves satisfy

(a) $\frac{c_{A}^{i^{\prime \prime}} q_{B A}}{c_{A}^{i^{\prime}}}=\frac{c_{B}^{i^{\prime \prime}} q_{B A}}{c_{B}^{i^{\prime}}}+1$,

(b) $c_{A}^{i^{\prime}}\left(1-\beta\left(1-\pi_{B}\right)\right)+q_{B A} c_{B}^{i^{\prime}}=0$.

Proof of Lemma 2. We use the short notation $c_{A}$ instead of $c_{A}^{i}$ and $u_{A}$ instead of $u_{i}\left(c_{A}^{i}\right)$ (the same for $c_{B}$ and $u_{B}$ ). Differentiate the two identities in (25) to arrive at

$$
\begin{aligned}
& u_{A}^{\prime} c_{A}^{\prime}+\frac{\beta \pi_{A}}{1-\beta\left(1-\pi_{B}\right)} u_{B}^{\prime} c_{B}^{\prime}=0, \\
& u_{A}^{\prime}+q_{B} u_{A}^{\prime \prime} c_{A}^{\prime}=\beta \pi_{A} u_{B}^{\prime \prime} c_{B}^{\prime} .
\end{aligned}
$$


Eq. (26) together with the Euler equation in (25) yields

$$
\left(1-\beta\left(1-\pi_{B}\right)\right) c_{A}^{\prime}+q_{B A} c_{B}^{\prime}=0,
$$

which proves part (b). Differentiate (26) again to obtain

$$
u_{A}^{\prime \prime}\left(c_{A}^{\prime}\right)^{2}+u_{A}^{\prime} c_{A}^{\prime \prime}+\frac{\beta \pi_{A}}{1-\beta\left(1-\pi_{B}\right)}\left(u_{B}^{\prime \prime}\left(c_{B}^{\prime}\right)^{2}+u_{B}^{\prime} c_{B}^{\prime \prime}\right)=0 .
$$

Inserting (27) gives

$$
\left(1-\beta\left(1-\pi_{B}\right)\right)\left(u_{A}^{\prime \prime}\left(c_{A}^{\prime}\right)^{2}+u_{A}^{\prime} c_{A}^{\prime \prime}\right)+c_{B}^{\prime} u_{A}^{\prime}+q_{B} u_{A}^{\prime \prime} c_{B}^{\prime} c_{A}^{\prime}+\beta \pi_{A} u_{B}^{\prime} c_{B}^{\prime \prime}=0 .
$$

Using (28) and the Euler equation again yields

$$
\left(1-\beta\left(1-\pi_{B}\right)\right) c_{A}^{\prime \prime}+c_{B}^{\prime}+c_{B}^{\prime \prime} q_{B A}=0 .
$$

Using (28) to replace $c_{B}^{\prime}$ in the second term and to replace $q_{B A}$ in the third term, and cancelling out the common factor $\left(1-\beta\left(1-\pi_{B}\right)\right)$ yields

$$
c_{A}^{\prime \prime}-\frac{c_{A}^{\prime}}{q_{B A}}-\frac{c_{A}^{\prime} c_{B}^{\prime \prime}}{c_{B}^{\prime}}=0 .
$$

Multiplying this expression by $q_{B A} / c_{A}^{\prime}$ proves part (a).

To complete the proof of Lemma 1, note that (24) holds if, and only if, this inequality is true for the aggregate consumption demands $C_{A}$ and $C_{B}$ for the agents in group $I_{A}$ instead of their excess demands $Z_{A}$ and $Z_{B}$. Using Lemma 2, formulas (a) and (b), we find that

$$
\begin{aligned}
\frac{C_{A}^{\prime \prime} q_{B A}}{C_{A}^{\prime}} & =\sum_{i \in I_{A}} \frac{c_{A}^{i^{\prime \prime}} q_{B A}}{c_{A}^{i^{\prime}}} \cdot \frac{c_{A}^{i^{\prime}}}{C_{A}^{\prime}} \\
& =\sum_{i \in I_{A}}\left(\frac{c_{B}^{i^{\prime \prime}} q_{B A}}{c_{B}^{i^{\prime}}}+1\right) \cdot \frac{c_{B}^{i^{\prime}}}{C_{B}^{\prime}} \\
& =\frac{C_{B}^{\prime \prime} q_{B A}}{C_{B}^{\prime}}+1 .
\end{aligned}
$$

This proves (24) and thus Lemma 1.

Proof of Proposition 2. Under the assumption of gross substitutes, the aggregate excess demand functions $Z_{A}^{B}$, etc. have the same features as in the proof of Proposition 1. In particular, the equilibrium conditions (MCA) and (MCB) define upward-sloping curves as depicted in Fig. 3. Again it only remains to show 1 and 2 as in the proof of Proposition 1.

On 1, we need to show that there is at most one intersection between (MCA) and (MCB). From the offer curve equation (15) we obtain by aggregation over all $i \in I_{A}$ that

$$
Z_{B}^{A}\left(q_{B A}\right) q_{B A}+\left(1-\beta\left(1-\pi_{B}\right)\right) Z_{A}^{A}\left(q_{B A}\right)=0 .
$$

Similarly, for the group $I_{B}$ :

$$
Z_{A}^{B}\left(q_{A B}\right) q_{A B}+\left(1-\beta\left(1-\pi_{A}\right)\right) Z_{B}^{B}\left(q_{A B}\right)=0 .
$$


Using these equations, we can rewrite (MCA) and (MCB) as

$$
\begin{aligned}
& Z_{A}^{A}\left(q_{B A}\right)-\frac{1-\beta\left(1-\pi_{A}\right)}{q_{A B}} Z_{B}^{B}\left(q_{A B}\right)=d, \\
& Z_{B}^{B}\left(q_{A B}\right)-\frac{1-\beta\left(1-\pi_{B}\right)}{q_{B A}} Z_{A}^{A}\left(q_{B A}\right)=d .
\end{aligned}
$$

Eliminating $Z_{B}^{B}$ gives

$$
Z_{A}^{A}\left(q_{B A}\right)\left(\frac{\left(1-\beta\left(1-\pi_{B}\right)\right)\left(1-\beta\left(1-\pi_{A}\right)\right)}{q_{B A}}-q_{A B}\right)+d\left(q_{A B}+1-\beta\left(1-\pi_{A}\right)\right)=0 .
$$

Because $Z_{A}^{A}<0$ is increasing in $q_{B A}$, the LHS of this equation is increasing in $q_{B A}$, and it is also increasing in $q_{A B}$. Thus, this equation defines a downward-sloping relationship between $q_{B} A$ and $q_{A B}$, whereas (MCA) and (MCB) define upward-sloping relations. Therefore, there can be at most one intersection between (MCA) and (MCB).

On 2, it is to show that the first best is an equilibrium if, and only if, there is no constrained equilibrium. The proof of this claim proceeds exactly as in the proof of Proposition 1. Note that $Z_{A}^{A}\left(q_{B A}^{*}\right)$ and $Z_{B}^{A}\left(q_{B A}^{*}\right)$ are excess demands of agents in group $I_{A}$ with the feature that the zerodebt constraint binds on all agents. Any first-best equilibrium must promise at least this level of consumption to agents in group $I_{A}$. Thus, when there is a first-best equilibrium with zero debt constraints, (23) holds, and vice versa. But this condition implies again that there cannot be an intersection between (MCA) and (MCB) above the line $q_{A B} q_{B A}=\beta^{2} \pi_{A} \pi_{B}$.

\section{References}

[1] F. Alvarez, U. Jermann, Efficiency, equilibrium and asset pricing with risk of default, Econometrica 68 (2000) $775-797$.

[2] T. Bewley, The optimum quantity of money, in: J. Karaken, N. Wallace (Eds.), Models of Monetary Economics, Federal Reserve Bank of Minneapolis, Minneapolis, 1980.

[3] J. Campbell, Consumption-based asset pricing, in: G. Constantinides, M. Harris, R. Stulz (Eds.), Handbook of the Economics of Finance, vol. 1B, North-Holland, Amsterdam, 2003, pp. 803-887.

[4] J. Campbell, J. Cochrane, By force of habit: a consumption-based explanation of aggregate stock market behavior, J. Polit. Economy 107 (1999) 205-251.

[5] G. Constantinides, Habit formation: a resolution of the equity premium puzzle, J. Polit. Economy 98 (1990) $519-543$.

[6] G. Constantinides, J. Donaldson, R. Mehra, Junior can't borrow: a new perspective of the equity premium puzzle, Quart. J. Econ. 117 (2002) 269-296.

[7] G. Constantinides, D. Duffie, Asset pricing with heterogeneous consumers, J. Polit. Economy 104 (1996) $219-240$.

[8] J. Heaton, D. Lucas, Evaluating the effects of incomplete markets on risk sharing and asset pricing, J. Polit. Economy 104 (1996) 443-487.

[9] T. Kehoe, D. Levine, Debt-constrained asset markets, Rev. Econ. Stud. 60 (1993) 865-888.

[10] T. Kehoe, D. Levine, Liquidity constrained markets versus debt constrained markets, Econometrica 69 (2001) 575-598.

[11] N. Kiyotaki, J. Moore, Credit cycles, J. Polit. Economy 105 (1997) 211-248.

[12] P. Krusell, A. Smith, Income and wealth heterogeneity in the macroeconomy, J. Polit. Economy 106 (1998) 867-896.

[13] H. Lustig, The market price of aggregate risk and the wealth distribution, NBER Working Paper No. $11132,2005$.

[14] N. Mankiw, S. Zeldes, The consumption of stockholders and nonstockholders, J. Finan. Econ. 29 (1991) 97-112.

[15] A. Sandroni, Asset prices and the distribution of wealth, Econ. Letters 64 (1999) 203-207.

[16] M. Santos, M. Woodford, Rational asset pricing bubbles, Econometrica 65 (1997) 19-57. 